\title{
Aplicação de vitamina C livre e encapsulada por spray chilling em salsicha de carne de frango: características físico-químicas, estabilidade e aceitação sensorial
}

\author{
Application of free or encapsulated vitamin $C$ to chicken frankfurter sausage by spray \\ chilling: physicochemical characteristics, stability and sensory acceptance
}

Autores | Authors

${ }^{*}$ Fernando Eustáquio de MATOS JUNIOR

Universidade de São Paulo (USP) Faculdade de Zootecnia e Engenharia de Alimentos

Departamento de Engenharia de Alimentos Av. Duque de Caxias Norte, 225 CEP: $13635-900$ Pirassununga/SP - Brasil e-mail:fematosjr@gmail.com

Marcelo THOMAZINI Marco Antonio TRINDADE Carmen Sílvia FÁVARO-TRINDADE

Universidade de São Paulo (USP)

Faculdade de Zootecnia e Engenharia de Alimentos

Departamento de Engenharia de Alimentos Pirassununga/SP - Brasil e-mail:mthomazini@usp.br trindadema@usp.br carmenft@usp.br

*Autor Correspondente / Corresponding Author

Recebido: Jul. 06, 2015

Aprovado: Jan. 29, 2016

\section{Resumo}

O aumento da conscientização do consumidor sobre a relação nutrição e saúde tem elevado os esforços para o desenvolvimento de alimentos contendo compostos bioativos capazes de promover benefícios adicionais à saúde além da nutrição básica, entre os quais a vitamina $\mathrm{C}$. Entretanto, muitas vezes a adição desses compostos pode ser prejudicada por sua baixa estabilidade, bem como pela possibilidade de alterar as características do produto ao qual são aplicados. O objetivo deste estudo foi avaliar o efeito da aplicação de vitamina C livre ou encapsulada nas características físico-químicas e sensoriais de salsicha de carne frango, além de avaliar sua estabilidade durante armazenamento refrigerado. Três tratamentos foram avaliados: salsicha com vitamina $C$ livre, salsicha com vitamina $C$ encapsulada por spray chilling e salsicha sem adição de vitamina C. As salsichas foram caracterizadas quanto aos seguintes parâmetros: estabilidade da emulsão, atividade de água, teor de umidade e teste de aceitação sensorial, com 60 provadores não treinados, em relação aos atributos de sabor, textura, cor, aroma e aceitabilidade global. As salsichas também foram avaliadas quanto à estabilidade durante armazenamento a $4^{\circ} \mathrm{C}$, por meio das análises de cor objetiva $\left(L^{*}, a^{*}\right.$ e $\left.b^{*}\right)$, valor de $\mathrm{pH}$, textura instrumental (dureza) e oxidação lipídica (TBARS). A aplicação da vitamina $C$, independentemente da forma aplicada, livre ou encapsulada, não alterou $(p>0,05)$ as características físico-químicas e sensoriais das salsichas, e promoveu estabilidade oxidativa satisfatória, mantendo os níveis de malonaldeído abaixo de 0,06 mg/kg de amostra durante 42 dias. Embora a encapsulação da vitamina $C$ não tenha demonstrado vantagens em relação à sua aplicação na forma livre, a utilização da vitamina $C$ livre nas concentrações estudadas neste trabalho, em salsicha produzida com carne de frango, como antioxidante, pode ser considerada promissora, tanto do ponto de vista tecnológico como para aumentar a saudabilidade deste produto.

Palavras-chave: Ácido ascórbico; Produto cárneo emulsionado; Substâncias reativas ao ácido tiobarbitúrico (TBARS); Encapsulação; Spray chilling.

\section{Summary}

The increase in consumer awareness about nutrition and health has increased the efforts to develop foods containing bioactive compounds that promote additional health benefits beyond the basic nutrition, and vitamin $\mathrm{C}$ is amongst these compounds. However, the addition of such compounds can be prejudiced by their low stability, or they could change the characteristics of the product to which they are applied. The aim of this study was to evaluate the effect of adding vitamin $\mathrm{C}$ in the free and encapsulated forms, on the physicochemical and sensory characteristics of chicken frankfurter sausage, and to evaluate its stability during cold storage. Three treatments were evaluated: sausage with free vitamin $C$, sausage with vitamin $C$ encapsulated by spray chilling and without vitamin $\mathrm{C}$. The sausages were characterized according to the emulsion stability, water activity, moisture content and sensory acceptance by 60 untrained panellists in relation to the following sensory attributes: taste, texture, colour, aroma and overall acceptance. The stability of the sausages was evaluated 
during their storage at $4{ }^{\circ} \mathrm{C}$ according to the instrumental colour $\left(\mathrm{L}^{*}, \mathrm{a}^{*}\right.$ and $\mathrm{b}^{*}$ parameters), $\mathrm{pH}$, instrumental texture (hardness) and lipid oxidation (TBARS). The application of vitamin $\mathrm{C}$, regardless of the form applied, free or encapsulated, did not change $(p>0.05)$ the physicochemical and sensory characteristics of the sausages promoting satisfactory oxidative stability while maintaining the levels of malondialdehyde below $0.06 \mathrm{mg} / \mathrm{kg}$ of sample for 42 days. Although the encapsulation of vitamin $\mathrm{C}$ showed no advantages in comparison to its application in the free form, its use in high concentrations as an antioxidant in chicken meat frankfurter sausages was promising, both from the technological point of view and to increase the healthfulness of this product.

Key words: Ascorbic acid; Emulsified meat product; Thiobarbituric acid-reactive substances (TBARS); Encapsulation; Spray chilling.

\section{Introdução}

O aumento da incidência das doenças crônicas não transmissíveis correlacionadas com o estilo de vida do homem contemporâneo e o aumento dos custos médico-hospitalares têm obrigado a sociedade a rever seus hábitos de vida. A alimentação, embora não seja o único fator capaz de comprometer o estado de saúde, é, sem dúvida, um dos mais importantes (JIMÉNEZ-COLMENERO et al., 2001). Com a crescente conscientização do impacto direto da alimentação no surgimento ou no agravo de doenças, uma série de novos comportamentos por parte do consumidor já pode ser constatada (OLIVEIRA et al., 2013; OLMEDILLA-ALONSO et al., 2013). Os consumidores estão buscando alimentos que, além de serem seguros, nutritivos, práticos e agradáveis ao paladar, também sejam capazes de promover bem-estar e prevenir doenças. Para atender a essa demanda, várias estratégias têm sido adotadas pela indústria de alimentos, entre as quais a redução e/ou exclusão de conservantes e corantes artificiais, a redução da concentração de sódio, a substituição de ingredientes cuja alta ingestão pode estar associada com o surgimento de patologias e a incorporação de compostos bioativos capazes de promover benefícios fisiológicos à saúde do consumidor. Essa última estratégia, em especial, tem apresentado um elevado crescimento, sendo que os alimentos que contêm os denominados compostos bioativos são chamados de alimentos funcionais (KHAN et al., 2011).

O desenvolvimento de alimentos funcionais tem crescido em todos os setores da indústria de alimentos, especialmente em produtos lácteos e cereais, com a incorporação de fibras, vitaminas antioxidantes, ácidos graxos funcionais e bactérias probióticas (BIGLIARDI; GALATI, 2013). Em todos os setores, o desenvolvimento de alimentos funcionais representa uma difícil tarefa, que exige sistemática cooperação dos diversos segmentos da indústria; porém, em nenhum outro setor, o desenvolvimento destes produtos é tão desafiador quanto no setor de produtos cárneos
(OLMEDILLA-ALONSO et al., 2013). Isso ocorre porque a relação dos consumidores com os produtos cárneos tem sido cada vez mais crítica, por conta da segurança nutricional desses produtos. Embora sejam alimentos altamente nutritivos e com elevada aceitação sensorial, a composição em ácidos graxos, o elevado teor de sódio e a presença de ingredientes, como sais de nitrito e nitrato de sódio, fazem com que o consumo de produtos cárneos seja associado com prejuízos à saúde, principalmente com o desencadeamento e/ou agravo de doenças cardiovasculares (REIG et al., 2006). Dessa forma, estratégias que visam a aumentar o apelo de "saudabilidade" dos produtos cárneos, seja pela incorporação de compostos bioativos e/ou alteração da composição, são indispensáveis para a desconstrução da imagem negativa relacionada a esses alimentos.

Diversos trabalhos vêm sendo desenvolvidos com a finalidade de incorporar compostos bioativos em produtos cárneos processados. Probióticos (RUBIO et al., 2014; DE VUYST et al., 2008; RUIZ et al., 2014), prebióticos (JIMÉNEZ-COLMENERO; DELGADO-PANDO, 2013), vitaminas (CÁCERES et al., 2008; JIMÉNEZ-COLMENERO et al., 2010), minerais (DE CIRIANO et al., 2010; NAVAS-CARRETERO et al., 2009) e antioxidantes com atividade funcional (SCHÖNFELDT; GIBSON, 2008; ZHANG et al., 2010; MUNEKATA et al., 2015) são exemplos de compostos bioativos que já foram incorporados em produtos cárneos com apelo de saudabilidade.

A vitamina C está entre os compostos bioativos que apresentam alto potencial para aplicação em produtos cárneos. Além das suas funções vitamínicas no organismo humano, sua função antioxidante contribuiu para prevenção da oxidação lipídica e dos pigmentos, preservando o sabor e a cor natural do produto cárneo (DAMODARAN et al., 2008). Apesar de suas diversas vantagens, devido às propriedades químicas da vitamina C, sua aplicação em determinados produtos cárneos, como os emulsionados, pode acarretar prejuízo da qualidade final do produto em função de alterações 
Aplicação de vitamina C livre e encapsulada por spray chilling em salsicha de carne de frango: características físico-químicas, estabilidade e aceitação sensorial

MATOS JUNIOR, F. E. et al.

causadas durante a etapa de processamento, como, por exemplo, comprometimento da estabilidade da emulsão por conta de possível redução excessiva do pH da massa cárnea (COMUNIAN et al., 2014). Acredita-se que esse problema poderia ser contornado com o uso da tecnologia de microencapsulação pela técnica de spray chilling, a qual tem por função isolar a vitamina $C$ durante o preparo da emulsão cárnea em micropartículas lipídicas que seriam derretidas durante o cozimento da salsicha, liberando a vitamina C para exercer sua função antioxidante.

Essa técnica, também designada como spray congealing, consiste da atomização de uma mistura, solução ou suspensão de material ativo e carreador fundido dentro de um ambiente mantido à temperatura abaixo do ponto de fusão do carreador. A atomização produz gotículas que se solidificam rapidamente ao entrar em contato com o ar frio, formando as micropartículas (OKURO et al., 2013b; FAVARO-TRINDADE et al., 2015). Em um estudo prévio, foi constatado que a microencapsulação por spray chilling promove proteção à vitamina $\mathrm{C}$, melhorando sua estabilidade em comparação com a sua forma não microencapsulada (MATOS-JUNIOR et al., 2015).

Diante do exposto, o objetivo deste trabalho foi avaliar o efeito de incorporação de vitamina C livre ou microencapsulada pela técnica de spray chilling nas características físico-químicas e sensoriais, e na estabilidade oxidativa de salsicha de carne de frango armazenada sob resfriamento.

\section{Material e métodos}

\subsection{Materiais}

Vitamina C (ácido ascórbico) de grau alimentício (Pantec, Rio de Janeiro/Brasil) e gordura interesterificada de palma e palmiste (Vigor, São Paulo/Brasil) foram utilizadas para produção das micropartículas lipídicas sólidas carregadas com vitamina C. Filé de coxa, sobrecoxa e pele de frango, sal de cura, sal ( $\mathrm{NaCl}$ ), pimenta branca em pó, cebola in natura e alho desidrato comerciais foram utilizados como ingredientes das salsichas.

\subsection{Produção das micropartículas lipídicas carregadas com ácido ascórbico}

As micropartículas lipídicas sólidas carregadas com vitamina $C$ foram produzidas através da técnica de spray chilling, que foi descrita por Okuro et al. (2013a), com algumas modificações. Para obtenção das micropartículas lipídicas, a gordura interesterificada de palma e palmiste foi fundida $43^{\circ} \mathrm{C}$, seguindo com adição da vitamina anidra. A atomização da mistura gordura e vitamina foi realizada em câmara fria a $13 \pm 2^{\circ} \mathrm{C}$, utilizando atomizador duplo fluido ( $\varnothing=1,2 \mathrm{~mm}$ ), com pressão de ar de 2,2 kgf $/ \mathrm{cm}^{2}$ em um spray chiller da Labmaq do Brasil (São Paulo/Brasil). A vazão foi controlada por meio de uma bomba peristáltica Masterflex (Illinois/EUA) em 50 LL/min. A proporção de vitamina em relação a gordura foi de 1:10, tendo sido escolhida em função de resultados obtidos em testes preliminares para obtenção da proporção ideal para melhoria significativa da estabilidade da vitamina. As micropartículas foram produzidas no dia anterior ao preparo das salsichas.

\subsection{Produção das salsichas de frango}

As salsichas foram produzidas a partir de formulações que atendessem aos Padrões de Identidade e Qualidade de Salsicha, determinados pelo Ministério da Agricultura, Pecuária e Abastecimento do Brasil (BRASIL, 2000). Definida a formulação padrão apresentada na Tabela 1, três tratamentos foram estabelecidos: salsicha com vitamina C livre (T1), salsicha com vitamina C microencapsulada (T2) e salsicha sem vitamina C (T3) (Tabela 2). As quantidades de vitamina $C$ livre ou encapsulada foram equivalentes.

Inicialmente, o filé de coxa e sobrecoxa e a pele foram moídos separadamente. Após pesagem, foram cominuídos e homogeneizados em homogeneizador rotativo de bacia (cutter) com os demais ingredientes. Retirada do cutter após atingir temperatura inferior a $14^{\circ} \mathrm{C}$, a massa foi embutida em tripas celulósicas com diâmetro de 22 mm, doadas pela Viscofan do Brasil (São Paulo/SP). Os gomos foram amarrados manualmente, mantendo o padrão de tamanho em torno de $15 \mathrm{~cm}$. Após embutidas, as salsichas foram acondicionadas adequadamente em carrinho tipo $\mathrm{H}$ e encaminhadas à estufa com vapor de água para a etapa de cozimento. O cozimento ocorreu até a temperatura de $72^{\circ} \mathrm{C}$ ser atingida no interior da salsicha, com duração aproximada de 1 hora e 10 minutos. Concluído o cozimento, as salsichas foram resfriadas por aspersão de água até atingir temperatura interna de $40^{\circ} \mathrm{C}$, conforme descrito por Uyhara et al. (2008), sendo então embaladas a vácuo (Ml 60, Selovac) em embalagens apropriadas para selagem a vácuo e estocadas em câmara fria a $4 \pm 1^{\circ} \mathrm{C}$.

\subsection{Caracterização físico-química da salsicha}

\subsubsection{Estabilidade da emulsão cárnea}

Após retirar a massa cárnea do cutter, aproximadamente $50 \mathrm{~g}$ de amostra foi reservada para análise de estabilidade da emulsão cárnea, realizada

Tabela 1. Formulação padrão para obtenção das salsichas elaboradas com carne de frango.

Ingrediente Quantidade (\%)

\begin{tabular}{cc} 
Filé de coxa e sobrecoxa de frango & 80 \\
Pele de frango & 10 \\
Água (gelo) & 10 \\
\hline
\end{tabular}


Aplicação de vitamina C livre e encapsulada por spray chilling em salsicha de carne de frango: características físico-químicas, estabilidade e aceitação sensorial

MATOS JUNIOR, F. E. et al.

Tabela 2. Composição de cada formulação (Tratamento) de salsichas adicionadas ou não de vitamina C livre ou encapsulada.

\begin{tabular}{lccc}
\multicolumn{1}{c}{ Ingrediente } & \multicolumn{1}{c}{ Formulação } \\
\cline { 2 - 4 } Vitamina C livre & T1 & T2 & T3 \\
Vitamina C encapsulada & $1000 \mathrm{ppm}$ & - & - \\
Cloreto de sódio (NaCl) & - & $1000 \mathrm{ppm}$ & $1,0 \%$ \\
Sal de cura & $1,0 \%$ & $1,0 \%$ & $0,5 \%$ \\
Pimenta branca em pó & $0,5 \%$ & $0,5 \%$ & $0,2 \%$ \\
Cebola & $0,2 \%$ & $0,2 \%$ & $2,0 \%$ \\
Alho desidratado & $2,0 \%$ & $2,0 \%$ & $0,5 \%$ \\
\hline
\end{tabular}

T1: Tratamento 1 com adição de vitamina C livre; T2: Tratamento 2 com adição de vitamina C encapsulada; T3: Tratamento 3 sem adição de vitamina C.

de acordo com a metodologia proposta por Parks e Carpenter (1987). As amostras foram acondicionadas em embalagens plásticas termorresistentes e seladas a vácuo. Após serem submetidas à pesagem, as amostras foram coccionadas em banho-maria a $70^{\circ} \mathrm{C}$ por 60 minutos. Findado o tempo de cocção, as embalagens foram abertas e o exsudato, descartado. Em seguida, as amostras foram novamente pesadas para avaliação da perda de massa.

\subsubsection{Atividade de água e umidade}

A atividade de água foi determinada com analisador de atividade de água Aqualab (Decagon Devices, Pullman, WA, USA), enquanto que a umidade foi determinada no analisador de umidade modelo MB35 Ohaus (Pine Brook, NJ, USA). As amostras de salsicha foram cortadas em pequenos pedaços e imediatamente introduzidas nos respectivos equipamentos para realização das análises. Ambas as análises foram realizadas 24 horas após a produção das salsichas, com as amostras resfriadas $\left(4 \pm 1^{\circ} \mathrm{C}\right)$. A análise de atividade de água foi realizada com equipamento indicando temperatura de $25^{\circ} \mathrm{C}$.

\subsubsection{Estabilidade das salsichas durante armazenamento refrigerado}

A estabilidade das salsichas foi avaliada em relação a cor objetiva, valor de $\mathrm{pH}$, dureza instrumental e índice de TBARS, nos tempos 1, 7, 14, 21, 28, 35 e 42 dias de estocagem refrigerada $\left(4^{\circ} \mathrm{C}\right)$.

\subsubsection{Valor de $\mathrm{pH}$}

As medições de $\mathrm{pH}$ foram realizadas com a utilização de um pHmetro (Modelo HI 99163, Marca HANNA), com eletrodo combinado para leitura em triplicata, com perfuração de três pontos de cada repetição.

\subsubsection{Análise de cor instrumental}

A alteração de cor das salsichas durante o período de estocagem foi investigada utilizando-se um colorímetro portátil (modelo Mini Scan XE Plus, marca HunterLab,
Reston, VA, USA). A cor foi expressa pelo sistema CIELab: $L^{*}$ (luminosidade), $a^{*}$ (intensidade de cor verde-vermelho) e $b^{\star}$ (intensidade de cor azul-amarelo). A análise foi realizada em triplicata, sendo tomadas três medidas de cada replicata em cada tratamento. As medidas foram realizadas na parte interna das amostras.

\subsubsection{Avaliação da dureza instrumental}

A dureza das amostras foi determinada por meio do texturômetro TA XT2i, (Stable Micro Systems, Godalming, Reino Unido), previamente calibrado com peso padrão de 2 kg. Para realização da análise, as salsichas foram cortadas em cilindros de tamanhos homogêneos, os quais foram comprimidos em até $70 \%$ do tamanho por um probe de alumínio (SMS P/20), com velocidade do pré-teste, teste e pós-teste de 2,0 mm/s e distância da plataforma de $16 \mathrm{~mm}$, em dez salsichas, à temperatura de $25^{\circ} \mathrm{C}$.

\subsubsection{Oxidação lipídica}

A estabilidade oxidativa das amostras de salsicha foi determinada utilizando-se o método de substâncias reativas ao ácido tiobarbitúrico (TBARS), segundo a metodologia de Vyncke (1970). O valor de TBARS foi calculado a partir de uma curva padrão e expresso em mg malonaldeído/kg do produto.

\subsubsection{Análise de aceitação sensorial}

A análise de aceitação sensorial foi realizada cinco dias após a produção da salsicha. Sessenta consumidores não treinados de ambos os sexos e diferentes faixas etárias participaram voluntariamente do teste. Todos os participantes foram inicialmente questionados se eram consumidores habituais de salsicha, sendo a resposta negativa um critério de exclusão. Os ensaios foram realizados em um laboratório de análise sensorial, em cabines individuais e conforme metodologia descrita por Meilgaard et al. (1999). As salsichas foram previamente aquecidas por cinco minutos em água fervente e cortadas em cilindros de $2 \mathrm{~cm}$, que foram mantidos submersos em água sob aquecimento em estufa a $40^{\circ} \mathrm{C}$. As amostras foram apresentadas monadicamente, em recipiente de 
Aplicação de vitamina C livre e encapsulada por spray chilling em salsicha de carne de frango: características físico-químicas, estabilidade e aceitação sensorial

MATOS JUNIOR, F. E. et al.

plástico codificado, e de forma aleatória. A avaliação foi realizada com escala hedônica de nove pontos (1 - desgostei muitíssimo e 9 - gostei muitíssimo) para os atributos de sabor, textura, cor, aroma e aceitabilidade global.

\subsection{Análise estatística}

Os dados foram analisados por ANOVA e as médias, comparadas pelo teste de Tukey no nível de significância de 5\%, com o auxílio do programa estatístico SAS (Statistic Analisy System), versão 9.2.

\section{Resultados e discussão}

\subsection{Caracterização físico-química da salsicha}

A adição de vitamina C livre ou microencapsulada não provocou alterações significativas $(p>0,05)$ na estabilidade das emulsões cárneas considerando-se os valores obtidos para o tratamento controle, em que a vitamina não foi adicionada.

Os valores percentuais de perda de massa dos três tratamentos variaram entre 18,27 \pm 0,64 e 19,74 $\pm 2,01$ (Tabela 3), sem diferença significativa $(p>0,05)$. Esses valores estão de acordo com os trabalhos consultados na literatura, entre os quais Nascimento et al. (2007), Yamada et al. (2010), Choi et al. (2011), Intarasirisawat et al. (2014) e Comunian et al. (2014), que reportaram valores de perda de massa que variavam de 1,4 0 0,2 a 17,19 $\pm 2,02$

A estabilidade da emulsão é utilizada como um indicador para avaliar a eficiência de retenção da gordura nas proteínas da carne (CHOI et al., 2011). Quando uma emulsão estável é obtida, não se verifica, após o cozimento do produto, separação da gordura dispersa. Diversos fatores podem influenciar a estabilidade da emulsão e, consequentemente, a obtenção do produto final.

Dentre os fatores que afetam a estabilidade da emulsão, o pH da massa durante o processo de emulsificação é considerado um ponto crítico (ARSLAN, 2006). Uma redução drástica de pH pode provocar desnaturação das proteínas solúveis do tecido muscular, inviabilizando o processo de emulsificação.
A adição de vitamina $C$ livre à massa cárnea poderia provocar tal ocorrência em função da redução rápida do pH; entretanto, a redução de pH causada nas salsichas pela adição da vitamina $\mathrm{C}$ livre e também da encapsulada não foi suficiente para influenciar negativamente no processo de emulsificação (Figura 1).

Os valores de atividade de água e umidade obtidos um dia após a produção das salsichas não apresentaram diferenças em função das formulações testadas $(p>0,05)$.

Os valores de umidade são importantes, sobretudo, para avaliar se o produto atende ao padrão de identidade e qualidade exigido pela legislação. No Brasil, esse padrão é regulamentado pelo Ministério da Agricultura e do Abastecimento, que determina que a salsicha não apresente umidade superior a $65 \%$. Assim, as salsichas produzidas neste trabalho atenderiam à legislação vigente (BRASIL, 2000). Todos os valores encontrados tanto para umidade quanto para atividade de água corroboram com valores citados na literatura, mantida a coerência em função das proporções utilizadas de cada ingrediente nas formulações (ANDRÉS et al., 2006; COMUNIAN et al., 2014; MENEGAS et al., 2013). Os valores de atividade de água (acima de 0,9) confirmam o quão perecível é a salsicha, representando um importante meio para proliferação de microrganismos indesejáveis quando o processamento e o armazenamento não ocorrem de maneira adequada.

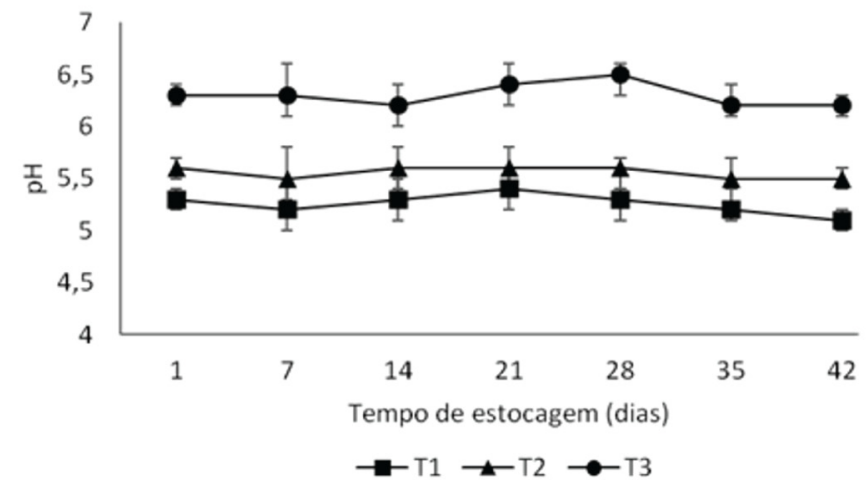

Figura 1. Valores de $\mathrm{pH}$ de salsichas analisados durante 42 dias de estocagem refrigerada. Tratamento 1: com adição de vitamina C livre; Tratamento 2: com adição de vitamina C encapsulada; Tratamento 3: sem adição de vitamina C.

Tabela 3. Estabilidade da emulsão cárnea durante o processamento e determinação da atividade de água e da umidade das salsichas após o processamento.

\begin{tabular}{cccc}
\hline Tratamento & Perda de massa (\%) & Atividade de água & Umidade (\%) \\
\hline Tratamento 1 & $19,14 \pm 1,24^{\mathrm{a}}$ & $0,966 \pm 0,004^{\mathrm{a}}$ & $55,36 \pm 3,73^{\mathrm{a}}$ \\
Tratamento 2 & $18,27 \pm 0,64^{\mathrm{a}}$ & $0,964 \pm 0,001^{\mathrm{a}}$ & $56,04 \pm 1,46^{\mathrm{a}}$ \\
Tratamento 3 & $19,74 \pm 2,01^{\mathrm{a}}$ & $0,964 \pm 0,003^{\mathrm{a}}$ & $58,67 \pm 1,12^{\mathrm{a}}$ \\
\hline
\end{tabular}

Médias na mesma coluna seguidas pela mesma letra não apresentam diferença significativa no nível de $5 \%$ de probabilidade pelo teste de Tukey, em que: Tratamento 1: com adição de vitamina C livre; Tratamento 2: com adição de vitamina C encapsulada; Tratamento 3: sem adição de vitamina C. 
Aplicação de vitamina C livre e encapsulada por spray chilling em salsicha de carne de frango: características físico-químicas, estabilidade e aceitação sensorial

MATOS JUNIOR, F. E. et al.

\subsection{Estabilidade das salsichas durante armazenamento refrigerado}

A estabilidade das salsichas pode ser avaliada em função do decaimento dos valores de $\mathrm{pH}$. No presente estudo, os valores de $\mathrm{pH}$ mantiveram-se constantes ( $p>0,05)$ em todos os tratamentos, durante os 42 dias.

A manutenção do pH foi satisfatória, visto que a redução do mesmo durante a estocagem pode predizer proliferação de bactérias láticas, que não apenas causam redução da qualidade sensorial do produto pelo gosto ácido, que se torna mais acentuado com aumento da atividade bacteriana, mas pela própria deterioração do produto.

Nas amostras provenientes do tratamento 3 (ausência de vitamina $\mathrm{C}$ ), foram verificados os maiores $(p<0,05)$ valores de $\mathrm{pH}(6,3 \pm 0,1)$. Não foram verificadas diferenças significativas $(p<0,05)$ entre os tratamentos com vitamina livre ou encapsulada, pois, em ambos os tratamentos, o $\mathrm{pH}$ ficou entre 5,3 \pm 0,1 e 5,5 \pm 0,2, respectivamente.

A salsicha sem adição de vitamina C, coerentemente, apresentou maior valor de $\mathrm{pH}$ em relação às demais.
Embora tenham sido constatadas diferenças entre o tratamento 3 e os demais, os valores de $\mathrm{pH}$ determinados encontram-se dentro da faixa $(5,0$ - 6,3) relatada por diversos autores que trabalharam com salsicha (ALESON-CARBONELL et al., 2003; RUIZ-MOYANO et al., 2011; SPAZIANI et al., 2009).

Os resultados da análise de cor instrumental, de acordo com os parâmetros $L^{*}, a^{*}, b^{*}$, são apresentados nas Tabelas 4, 5 e 6, respectivamente. Nenhum dos parâmetros avaliados apresentou diferenças entre os tratamentos e nem sofreu alterações significativas durante o período de estocagem. Valores muito semelhantes para os três parâmetros, $L^{*}, a^{*}, b^{*}$, foram relatados por Andrés et al. (2006), que avaliaram a estabilidade de salsichas de frango com baixo teor de gordura durante o armazenamento. Comunian et al. (2014), que também avaliaram a adição de vitamina $C$ em salsicha de frango, encontraram valores próximos, com discreta elevação do parâmetro b*, o qual avalia a variação do azul (-) ao amarelo (+). No tratamento (T3), que sofreu acentuada oxidação lipídica, confirmada pela análise de TBARS, poderia ter sido constatada alteração de cor, em

Tabela 4. Valores de $L^{*}$ para as três formulações de salsichas de frango com e sem adição de vitamina C, durante 42 dias de armazenamento.

\begin{tabular}{cccccccc}
\multirow{2}{*}{ Tratamentos } & \multicolumn{7}{c}{ Tempo de estocagem refrigerada (dias) } \\
\cline { 2 - 8 } & $\mathbf{1}$ & $\mathbf{7}$ & $\mathbf{1 4}$ & $\mathbf{2 1}$ & $\mathbf{2 8}$ & $\mathbf{3 5}$ & $\mathbf{4 2}$ \\
\hline T1 & $67,47 \pm 1,24^{\mathrm{aA}}$ & $68,87 \pm 2,04^{\mathrm{aA}}$ & $68,41 \pm 0,91^{\mathrm{aA}}$ & $68,03 \pm 1,19^{\mathrm{aA}}$ & $68,83 \pm 0,98^{\mathrm{aA}}$ & $69,14 \pm 1,07^{\mathrm{aA}}$ & $69,99 \pm 1,81^{\mathrm{aA}}$ \\
$\mathrm{T}_{2}$ & $69,33 \pm 1,87^{\mathrm{aA}}$ & $70,73 \pm 1,02^{\mathrm{aA}}$ & $70,24 \pm 0,94^{\mathrm{aA}}$ & $71,74 \pm 1,13^{\mathrm{aA}}$ & $71,05 \pm 1,24^{\mathrm{aA}}$ & $71,5 \pm 1,67^{\mathrm{aA}}$ & $71,79 \pm 1,47^{\mathrm{aA}}$ \\
$\mathrm{T}_{3}$ & $69,57 \pm 1,56^{\mathrm{aA}}$ & $71,57 \pm 0,80^{\mathrm{aA}}$ & $71,48 \pm 2,01^{\mathrm{aA}}$ & $71,25 \pm 1,24^{\mathrm{aA}}$ & $70,59 \pm 1,44^{\mathrm{aA}}$ & $70,98 \pm 0,76^{\mathrm{aA}}$ & $70,21 \pm 0,56^{\mathrm{aA}}$ \\
\hline
\end{tabular}

Médias seguidas por uma mesma letra minúscula na mesma coluna e letra maiúscula na mesma linha não diferem estatisticamente pelo teste de Tukey no nível de 5\% de probabilidade, em que: Tratamento 1: com adição de vitamina C livre; Tratamento 2: com adição de vitamina C encapsulada; Tratamento 3: sem adição de vitamina C.

Tabela 5. Valores de $a^{*}$ para as três formulações de salsichas de frango com e sem adição de vitamina C, durante 42 dias de armazenamento.

\begin{tabular}{cccccccc}
\multirow{2}{*}{ Tratamentos } & \multicolumn{7}{c}{ Tempo de estocagem refrigerada (dias) } \\
\cline { 2 - 7 } & $\mathbf{1}$ & $\mathbf{7}$ & $\mathbf{1 4}$ & $\mathbf{2 1}$ & $\mathbf{2 8}$ & $\mathbf{3 5}$ & $\mathbf{4 2}$ \\
T1 & $3,78 \pm 1,01^{\mathrm{aA}}$ & $3,50 \pm 1,22^{\mathrm{aA}}$ & $3,71 \pm 2,02^{\mathrm{aA}}$ & $3,95 \pm 1,87^{\mathrm{aA}}$ & $4,02 \pm 2,42^{\mathrm{aA}}$ & $4,16 \pm 1,52^{\mathrm{aA}}$ & $4,20 \pm 1,94^{\mathrm{aA}}$ \\
$\mathrm{T} 2$ & $3,84 \pm 1,64^{\mathrm{aA}}$ & $3,86 \pm 0,86^{\mathrm{aA}}$ & $3,79 \pm 1,74^{\mathrm{aA}}$ & $3,76 \pm 1,69^{\mathrm{aA}}$ & $3,83 \pm 1,97^{\mathrm{aA}}$ & $4,06 \pm 0,83^{\mathrm{aA}}$ & $4,18 \pm 1,67^{\mathrm{aA}}$ \\
T3 & $3,61 \pm 1,42^{\mathrm{aA}}$ & $3,73 \pm 1,81^{\mathrm{Aa}}$ & $3,91 \pm 0,94^{\mathrm{aA}}$ & $3,67 \pm 1,05^{\mathrm{aA}}$ & $3,41 \pm 1,56^{\mathrm{aA}}$ & $3,69 \pm 1,64^{\mathrm{aA}}$ & $3,75 \pm 1,32^{\mathrm{aA}}$ \\
\hline
\end{tabular}

Médias seguidas por uma mesma letra minúscula na mesma coluna e letra maiúscula na mesma linha não diferem estatisticamente pelo teste de Tukey no nível de $5 \%$ de probabilidade, em que: Tratamento 1: com adição de vitamina C livre; Tratamento 2: com adição de vitamina C encapsulada; Tratamento 3: sem adição de vitamina C.

Tabela 6. Valores de b* para as três formulações de salsichas de frango com e sem adição de vitamina C, durante 42 dias de armazenamento.

\begin{tabular}{|c|c|c|c|c|c|c|c|}
\hline \multirow{2}{*}{ Tratamentos } & \multicolumn{7}{|c|}{ Tempo de estocagem refrigerada (dias) } \\
\hline & 1 & 7 & 14 & 21 & 28 & 35 & 42 \\
\hline T1 & $11,24 \pm 0,84^{\mathrm{aA}}$ & $12,05 \pm 1,37^{\mathrm{aA}}$ & $12,08 \pm 0,69^{a A}$ & $12,65 \pm 1,28^{\mathrm{aA}}$ & $10,91 \pm 1,52^{\mathrm{aA}}$ & $11,69 \pm 1,11^{\mathrm{aA}}$ & $11,15 \pm 0,96^{a A}$ \\
\hline T2 & $12,91 \pm 1,15^{\mathrm{aA}}$ & $13,65 \pm 0,91^{\mathrm{aA}}$ & $12,25 \pm 1,68^{\mathrm{aA}}$ & $11,41 \pm 0,94^{\mathrm{aA}}$ & $11,34 \pm 2,61^{\mathrm{aA}}$ & $12,51 \pm 1,67^{\mathrm{aA}}$ & $11,28 \pm 1,37^{\mathrm{aA}}$ \\
\hline T3 & $11,40 \pm 1,67^{\mathrm{aA}}$ & $12,57 \pm 1,23^{\mathrm{aA}}$ & $11,94 \pm 1,92^{\mathrm{aA}}$ & $12,18 \pm 1,67^{\mathrm{aA}}$ & $11,10 \pm 1,81^{\mathrm{aA}}$ & $12,61 \pm 1,84^{\text {aA }}$ & $12,22 \pm 0,72^{\mathrm{aA}}$ \\
\hline
\end{tabular}

Médias seguidas por uma mesma letra minúscula na mesma coluna e letra maiúscula na mesma linha não diferem estatisticamente pelo teste de Tukey no nível de 5\% de probabilidade, em que: Tratamento 1: com adição de vitamina C livre; Tratamento 2: com adição de vitamina C encapsulada; Tratamento 3: sem adição de vitamina C 
Aplicação de vitamina C livre e encapsulada por spray chilling em salsicha de carne de frango: características físico-químicas, estabilidade e aceitação sensorial

MATOS JUNIOR, F. E. et al.

especial nos valores de $a^{*}$, devida à possível oxidação do pigmento mioglobina; porém essa alteração não foi verificada.

Como apresentado na Tabela 7, a adição da vitamina C (livre ou encapsulada) não alterou a dureza da salsicha. Alguma alteração poderia ter ocorrido em função do abaixamento do valor de $\mathrm{pH}$ causado pela adição de vitamina C (Figura 1), que poderia ter alterado a funcionalidade das proteínas cárneas em função de uma maior aproximação do seu ponto isoelétrico (DAMODARAN et al., 2008). No entanto, assim como verificado nas análises de estabilidade das emulsões, não ocorreram alterações neste sentido.

A elevada quantidade de proteínas de boa qualidade adicionada nas formulações desenvolvidas (80\% de filé de coxa e sobrecoxa de frango) pode ter contribuído para a garantia de uma emulsão firme e estável mesmo com o menor pH. Além disso, durante o período de estocagem, não foi constatada alteração da textura em nenhum tratamento ( $p>0,05)$.

As concentrações de malonaldeído determinadas durante o período de estocagem refrigerada são apresentadas na Figura 2. Não foram constatadas diferenças $(p>0,05)$ entre as amostras que receberam

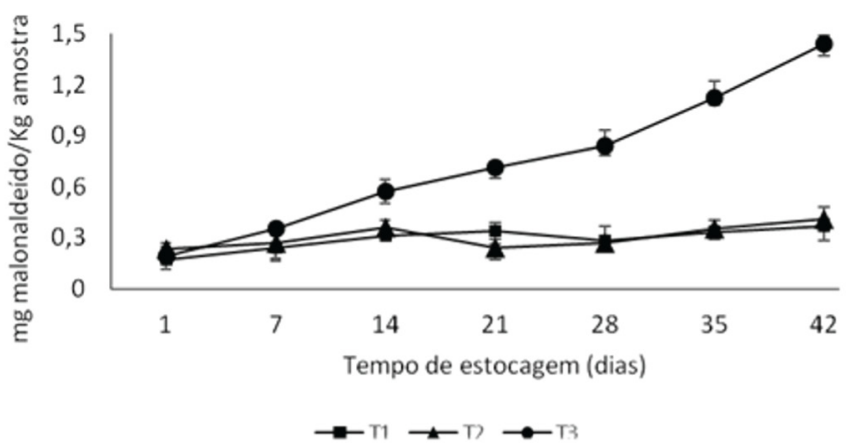

Figura 2. Valores de TBARS (mg malonaldeído/kg amostra) para cada tratamento, durante 42 dias de estocagem refrigerada. Tratamento 1: com adição de vitamina C livre; Tratamento 2: com adição de vitamina C encapsulada; Tratamento 3: sem adição de vitamina $\mathrm{C}$. a vitamina $\mathrm{C}$ livre ou encapsulada em cada tempo e ao longo do período de estocagem. Este resultado permite inferir que, independentemente da forma como o aditivo foi incorporado à massa, este se mostrou efetivo no controle da oxidação do produto. Na amostra controle - sem adição de vitamina C - a concentração de malonaldeído foi maior $(p<0,05)$ em relação às demais já com sete dias de estocagem, aumentando sua concentração $(p<0,05)$ durante a estocagem, indicando que o produto estava sofrendo o processo de oxidação.

A oxidação lipídica das salsichas pode ser relacionada com a quantidade de lipídios, em especial fosfolipídios e ácidos graxos insaturados, presentes nos produtos. A carne de aves, devido ao seu elevado conteúdo de ácidos graxos insaturados (em torno de $70 \%$ ), é altamente susceptível à oxidação (MURPHY et al., 2004).

\subsection{Análise de aceitação sensorial}

As médias das notas atribuídas pelos consumidores na análise sensorial da salsicha são apresentadas na Tabela 8. Não houve diferença significativa entre os tratamentos em nenhum dos atributos avaliados e isso significa que a adição de vitamina $C$, tanto livre quanto encapsulada, não provocou alterações na aceitação sensorial das salsichas. Entre os atributos, a cor foi a que recebeu as menores notas. Esses resultados são coerentes, considerando-se que não houve adição de corante nas formulações estudadas. Os participantes da análise eram consumidores de salsicha; assim, utilizaram suas experiências prévias como parâmetro para avaliação, sendo que salsichas comercializadas no Brasil normalmente apresentam corante em sua formulação.

Resultados semelhantes já foram descritos por outros autores, como Uyhara et al. (2008) e Moreira et al. (2008), que desenvolveram salsichas com filé de tilápia com e sem corante. A textura foi outro atributo cuja avaliação pode ter sido influenciada por experiências prévias. A quantidade de carne presente na formulação é um dos fatores que mais influenciam a textura do produto final. A quantidade de carne presente nas formulações

Tabela 7. Valores de dureza de salsichas de frango com e sem adição de vitamina C, durante estocagem refrigerada.

\begin{tabular}{cccc} 
Tempo (dias) & \multicolumn{3}{c}{ Dureza (g) } \\
\cline { 2 - 4 } 1 & $4347,20 \pm 614,21^{\mathrm{aA}}$ & $4447,24 \pm 542,01^{\mathrm{aA}}$ & \multicolumn{1}{c}{ T3 } \\
7 & $4411,34 \pm 479,11^{\mathrm{aA}}$ & $4365,83 \pm 671,24^{\mathrm{aA}}$ & $4459,512 \pm 128,45^{\mathrm{aA}}$ \\
14 & $4449,74 \pm 371,55^{\mathrm{aA}}$ & $4278,08 \pm 248,54^{\mathrm{aA}}$ & $4407,349 \pm 514,30^{\mathrm{aA}}$ \\
21 & $4298,11 \pm 1241,2^{\mathrm{aA}}$ & $4377,64 \pm 954,01^{\mathrm{aA}}$ & $4384,544 \pm 421.02^{\mathrm{aA}}$ \\
28 & $4426,09 \pm 475,32^{\mathrm{aA}}$ & $4417,44 \pm 174,28^{\mathrm{aA}}$ & $4459,675 \pm 646,52^{\mathrm{aA}}$ \\
35 & $4287,84 \pm, 074,36^{\mathrm{aA}}$ & $4409,34 \pm 254,37^{\mathrm{aA}}$ & $4285,844 \pm 516,58^{\mathrm{aA}}$ \\
42 & $4293,38 \pm 924,07^{\mathrm{aA}}$ & $4311,17 \pm 484,25^{\mathrm{aA}}$ & $4452,682 \pm 184,24^{\mathrm{aA}}$ \\
\hline
\end{tabular}

Médias seguidas por uma mesma letra minúscula na mesma coluna e letra maiúscula na mesma linha não diferem estatisticamente pelo teste de Tukey no nível de $5 \%$ de probabilidade, em que: Tratamento 1: com adição de vitamina C livre; Tratamento 2: com adição de vitamina C encapsulada; Tratamento 3: sem adição de vitamina C. 
Aplicação de vitamina C livre e encapsulada por spray chilling em salsicha de carne de frango: características físico-químicas, estabilidade e aceitação sensorial

MATOS JUNIOR, F. E. et al.

Tabela 8. Médias dos valores obtidos para cada atributo (aroma, cor, sabor, textura e aceitação global) na avaliação sensorial de salsicha de frango com ou sem vitamina $\mathrm{C}$.

\begin{tabular}{cccccc}
\hline Tratamento & Aroma & Cor & Sabor & Textura & AG \\
T1 & $7,21 \pm 1,31^{\mathrm{a}}$ & $4,61 \pm 1,45^{\mathrm{a}}$ & $6,73 \pm 1,22^{\mathrm{a}}$ & $5,27 \pm 1,36^{\mathrm{a}}$ & $6,72 \pm 1,63^{\mathrm{a}}$ \\
T2 & $7,43 \pm 1,15^{\mathrm{a}}$ & $4,68 \pm 1,84^{\mathrm{a}}$ & $6,67 \pm 1,54^{\mathrm{a}}$ & $5,46 \pm 1,41^{\mathrm{a}}$ & $6,61 \pm 1,24^{\mathrm{a}}$ \\
T3 & $7,17 \pm 1,57^{\mathrm{a}}$ & $4,97 \pm 1,61^{\mathrm{a}}$ & $6,84 \pm 1,09^{\mathrm{a}}$ & $5,64 \pm 1,29^{\mathrm{a}}$ & $6,85 \pm 1,73^{\mathrm{a}}$ \\
\hline
\end{tabular}

$\mathrm{AG}=$ Avaliação global. Médias na mesma coluna seguidas pela mesma letra não apresentam diferença significativa no nível de $5 \%$ de probabilidade pelo teste de Tukey, em que: Tratamento 1: com adição de vitamina C livre; Tratamento 2: com adição de vitamina C encapsulada; Tratamento 3: sem adição de vitamina C.

avaliadas é superior em relação às formulações das salsichas comercializadas no Brasil, que normalmente utilizam alto percentual de carne mecanicamente separada de aves, fazendo com que a textura realmente seja mais resistente.

Como não foi detectada diferença significativa entre os tratamentos para o atributo sabor, é possível concluir que a diferença de $\mathrm{pH}$ detectada (Figura 2) não alterou significativamente este atributo e, portanto, não comprometeu a aceitação sensorial dos produtos elaborados com a vitamina C nas formas livre ou encapsulada. Os atributos aroma e sabor receberam avaliações de "gostei regularmente" e "gostei ligeiramente", respectivamente. A utilização de cebola in natura e alho desidratado conferiram ao produto aroma e sabor marcantes, o que certamente favoreceu as notas para ambos os atributos. A aceitação global para todos os tratamentos ficou com média 6 (gostei ligeiramente), resultado semelhante ao encontrado por Vogel et al. (2011) e Comunian et al. (2014). A incorporação de um corante natural poderia melhorar as notas para o atributo cor e, consequentemente, poderia melhorar os resultados de aceitação geral.

\section{Conclusão}

A adição de vitamina $C$ em salsicha de frango com o objetivo de promover suplementação dietética deste nutriente e, ao mesmo tempo, utilizá-lo com agente antioxidante, preservando a estabilidade oxidativa do produto, foi bem-sucedida no presente trabalho. Os resultados demonstraram que a adição de 1000 ppm da vitamina não promoveu alterações durante o processamento do produto e não influenciou nas características físico-químicas do produto armazenado sob resfriamento durante 60 dias ou nas suas características sensoriais. A adição da vitamina encapsulada não demonstrou vantagem em relação à sua forma livre. Em estudos futuros, a eficácia da encapsulação em concentrações maiores de vitamina pode ser testada.

\section{Agradecimentos}

Os autores agradecem à Fundação de Amparo à Pesquisa do Estado de São Paulo (FAPESP) pela Bolsa de Estudos concedida (Processo 2010/13117-5).

\section{Referências}

ALESON-CARBONELL, L.; FERNANDEZ-LOPEZ, J.; SAYASBARBERA, E.; SENDRA, E.; PEREZ-ALVAREZ, J. A. Utilization of lemon albedo in dry-cured sausages. Journal of Food Science, Malden, v. 68, n. 5, p. 1826-1830, 2003. http://dx.doi. org/10.1111/j.1365-2621.2003.tb12337.x.

ANDRÉS, S. C.; GARCÍA, M. E.; ZARITZKY, N. E.; CALIFANO, A. N. Storage stability of low-fat chicken sausages. Journal of Food Engineering, Pullman, v. 72, n. 4, p. 311-319, 2006. http:// dx.doi.org/10.1016/j.jfoodeng.2004.08.043.

ARSLAN, S. Effects of salt and phosphate levels on the emulsion properties of fresh and frozen hen meats. African Journal of Biotechnology, Lagos, v. 5, n. 10, p. 86-89, 2006.

BIGLIARDI, B.; GALATI, F. Innovation trends in the food industry: the case of functional foods. Trends in Food Science \& Technology, Oxford, v. 31, n. 2, p. 118-129, 2013. http://dx.doi. org/10.1016/j.tifs.2013.03.006.

BRASIL. Ministério da Agricultura e do Abastecimento. Secretaria de Defesa Agropecuária. Instrução Normativa $n^{\circ} 4$, de 31 de março de 2000. Aprova os regulamentos técnicos de identidade e qualidade de carne mecanicamente separada, de mortadela, de linguiça e de salsicha. Diário Oficial [da] República Federativa do Brasil, Brasília, DF, 05 abr. 2000. Seção 1, v. 1, p. 6-10.

CÁCERES, E.; GARCÍA, M.; SELGAS, M. Conventional and reduced-fat cooked meat sausages enriched with folic acid. Fleischwirtschaft, Frankfurt, v. 23, p. 58-60, 2008.

CHOI, Y.-S.; KIM, H.-W.; SONG, D.-H.; CHO, J.-H.; PARK, J.-H.; KIM, M.-Y.; LIM, C.-S.; KIM, C.-J. Quality characteristics and sensory properties of reduced-fat emulsion sausages with brown rice fiber. Korean Journal for Food Science of Animal Resources, Gangnam-gu, v. 31, n. 4, p. 521-529, 2011. http:// dx.doi.org/10.5851/kosfa.2011.31.4.521.

COMUNIAN, T. A.; THOMAZINI, M.; GAMBAGORTE, V. F.; TRINDADE, M. A.; FAVARO-TRINDADE, C. S. Effect of incorporating free or encapsulated ascorbic acid in chicken frankfurters on physicochemical and sensory stability. Journal of Food Science and Engineering, Libertyville, v. 4, p. 167-175, 2014.

DAMODARAN, S.; PARKIN, K. L.; FENNEMA, O. R. Fennema's food chemistry. Boca Raton: CRC Press, 2008. 
Aplicação de vitamina C livre e encapsulada por spray chilling em salsicha de carne de frango: características físico-químicas, estabilidade e aceitação sensorial

MATOS JUNIOR, F. E. et al.

DE CIRIANO, M. G-I.; LAREQUI, E.; REHECHO, S.; CALVO, M. I.; CAVERO, R. Y.; NAVARRO-BLASCO, Í.; ASTIASARÁN, I.; ANSORENA, D. Selenium, iodine, $\omega-3$ PUFA and natural antioxidant from Melissa officinalis L.: a combination of components from healthier dry fermented sausages formulation. Meat Science, Barking, v. 85, n. 2, p. 274-279, 2010. http:// dx.doi.org/10.1016/j.meatsci.2010.01.012. PMid:20374898.

DE VUYST, L.; FALONY, G.; LEROY, F. Probiotics in fermented sausages. Meat Science, Barking, v. 80, n. 1, p. 75-78, 2008. http://dx.doi.org/10.1016/j.meatsci.2008.05.038. PMid:22063171.

FAVARO-TRINDADE, C. S.; OKURO, P. K.; MATOS-JR, F. E. Encapsulation via Spray Chilling/Cooling/Congealing. In: MISHRA, M. K. (Org.). Handbook of encapsulation and controlled release. Boca Raton: CRC Press, 2015. v. 1, p. 71-88.

INTARASIRISAWAT, R.; BENJAKUL, S.; VISESSANGUAN, W.; $W U, J$. Effects of skipjack roe protein hydrolysate on properties and oxidative stability of fish emulsion sausage. LWT - Food Science and Technology, Oxford, v. 58, n. 1, p. 280-286, 2014. JIMÉNEZ-COLMENERO, F.; CARBALLO, J.; COFRADES, S. Healthier meat and meat products: their role as functional foods. Meat Science, Barking, v. 59, n. 1, p. 5-13, 2001. http://dx. doi. org/10.1016/S0309-1740(01)00053-5. PMid:22062500.

JIMÉNEZ-COLMENERO, F.; DELGADO-PANDO, G. Fibreenriched meat products. In: DELCOUR, J.; POUTANEN, K. (Ed.). Fibre-rich and wholegrain foods. Cambridge: Woodhead Publishing Limited, 2013. p. 329-347.

JIMÉNEZ-COLMENERO, F.; SÁNCHEZ-MUNIZ, F. J.; OLMEDILLAALONSO, B. Design and development of meat-based functional foods with walnut: technological, nutritional and health impact. Food Chemistry, London, v. 123, n. 4, p. 959-967, 2010. http:// dx.doi.org/10.1016/j.foodchem.2010.05.104.

KHAN, M. I.; ARSHAD, M. S.; ANJUM, F. M.; SAMEEN, A.; ANEEQ-UR-REHMAN; GILL, W. T. Meat as a functional food with special reference to probiotic sausages. Food Research International, Oxford, v. 44, n. 10, p. 3125-3133, 2011. http:// dx.doi.org/10.1016/j.foodres.2011.07.033.

MATOS-JUNIOR, F. E.; DI SABATINO, M.; PASSERINI, N.; FAVARO-TRINDADE, C. S.; ALBERTINI, B. Development and characterization of solid lipid microparticles loaded with ascorbic acid and produced by spray congealing. Food Research International, Oxford, v. 67, p. 52-59, 2015. http://dx.doi. org/10.1016/j.foodres.2014.11.002.

MEILGAARD, M.; CIVILLE, G.; CARR, B. Sensory evaluation techniques. 3. ed. New York: CRC Press, 1999.

MENEGAS, L. Z.; PIMENTEL, T. C.; GARCIA, S.; PRUDENCIO, S. H. Dry-fermented chicken sausage produced with inulin and corn oil: physicochemical, microbiological, and textural characteristics and acceptability during storage. Meat Science,
Barking, v. 93, n. 3, p. 501-506, 2013. http://dx.doi.org/10.1016/j. meatsci.2012.11.003. PMid:23273457.

MOREIRA, R. T.; LEMOS, A. L. S. C.; HARADA, M. M.; CIPOLLI, K.; MENDES, E. S.; GUIMARÃES, J. L.; CRISTIANINI, M. Desenvolvimento e aceitação de embutido emulsionado tipo mortadela elaborado com tilápia (Oreochromis niloticus L.). Higiene Alimentar, São Paulo, v. 159, n. 22, p. 47-52, 2008.

MUNEKATA, P. E.; CALOMENI, A. V.; RODRIGUES, C. E.; FÁVARO-TRINDADE, C. S.; ALENCAR, S. M.; TRINDADE, M. A. Peanut skin extract reduces lipid oxidation in cooked chicken patties. Poultry Science, Oxford, v. 94, n. 3, p. 442-446, 2015. http://dx.doi.org/10.3382/ps/pev005. PMid:25681473.

MURPHY, S. C.; GILROY, D.; KERRY, J. F.; BUCKLEY, D. J.; KERRY, J. P. Evaluation of surimi, fat and water content in a low/no added pork sausage formulation using response surface methodology. Meat Science, Barking, v. 66, n. 3, p. 689-701, 2004. http://dx.doi.org/10.1016/j.meatsci.2003.07.001. PMid:22060879.

NASCIMENTO, R.; CAMPAGNOL, P. C. B.; MONTEIRO, E. S.; POLLONIO, M. A. R. Substituição de cloreto de sódio por cloreto de potássio: influência sobre as características físico-químicas e sensoriais de salsichas. Revista de Alimentos e Nutrição, Araraquara, v. 18, n. 3, p. 297-302, 2007

NAVAS-CARRETERO, S.; PÉREZ-GRANADOS, A. M.; SARRIÁ, B.; VAQUERO, M. P. Iron absorption from meat pate fortified with ferric pyrophosphate in iron-deficient women. Nutrition, London, v. 25, n. 1, p. 20-24, 2009. http://dx.doi.org/10.1016/j. nut.2008.07.002. PMid:18752926.

OKURO, P. K.; THOMAZINI, M.; BALIEIRO, J. C. C.; LIBERAL, R. D. C. O.; FAVARO-TRINDADE, C. S. Co-encapsulation of Lactobacillus acidophilus with inulin or polydextrose in solid lipid microparticles provides protection and improves stability. Food Research International, Barking, v. 53, n. 1, p. 96-103, 2013 a. http://dx.doi.org/10.1016/j.foodres.2013.03.042.

OKURO, P. K.; MATOS-JUNIOR, F. E.; FAVARO-TRINDADE, C. S. Technological challenges for spray chilling encapsulation of functional food ingredients. Food Technology and Biotechnology, Zagreb, v. 51, n. 2, p. 171-182, 2013b.

OLIVEIRA, D. F.; COELHO, A. R.; BURGARDT, V. C. F.; HASHIMOTO, E. H.; LUNKES, A. M.; MARCHI, J. F.; TONIAL, I. B. Alternativas para um produto cárneo mais saudável: uma revisão. Brazilian Journal of Food Technology, Campinas, v. 16, ก. 3, 2013.

OLMEDILLA-ALONSO, B.; JIMÉNEZ-COLMENERO, F.; SÁNCHEZ-MUNIZ, F. J. Development and assessment of healthy properties of meat and meat products designed as functional foods. Meat Science, Barking, v. 95, n. 4, p. 919-930, 2013. http:// dx.doi.org/10.1016/j.meatsci.2013.03.030. PMid:23623320.

PARKS, L. L.; CARPENTER, J. A. Functionality of six non meat proteins in meat emulsion systems. Journal of Food 
Aplicação de vitamina C livre e encapsulada por spray chilling em salsicha de carne de frango: características físico-químicas, estabilidade e aceitação sensorial

MATOS JUNIOR, F. E. et al.

Science, Chicago, v. 52, n. 2, p. 271-274, 1987. http://dx.doi. org/10.1111/j.1365-2621.1987.tb06590.x.

REIG, M.; TOLDRÁ, F.; JIMÉNEZ-COLMENERO, F. New approaches for the development of functional meat products. In: NOLLET, L. M. L.; TOLDRÁ, F. (Ed.). Advanced technologies for meat processing. Boca Raton: CRC Press, 2006. p. 275-308.

RUBIO, R.; JOFRÉ, A.; AYMERICH, T.; GUÀRDIA, M. D.; GARRIGA, M. Nutritionally enhanced fermented sausages as a vehicle for potential probiotic lactobacilli delivery. Meat Science, Barking, v. 96, n. 2 Pt A, p. 937-942, 2014. http://dx. doi. org/10.1016/j.meatsci.2013.09.008. PMid:24211552.

RUIZ, J. N.; VILLANUEVA, N. D. M.; FAVARO-TRINDADE, C. S.; CONTRERAS-CASTILLO, C. J. Physicochemical, microbiological and sensory assessments of Italian salami sausages with probiotic potential. Scientia Agricola, Piracicaba, v. 71, n. 3, p. 204-211, 2014. http://dx.doi.org/10.1590/S010390162014000300005.

RUIZ-MOYANO, S.; MARTÍN, A.; BENITO, M. J.; ARANDA, E.; CASQUETE, R.; CÓRDOBA, M. G. Implantation ability of the potential probiotic strain Lactobacillus reuteri PL519, in "Salchichón," a traditional iberian dry fermented sausage. Journal of Food Science, Chicago, v. 76, n. 5, p. M268-M275, 2011. http://dx.doi.org/10.1111/j.1750-3841.2011.02189.x. PMid:22417437.

SCHÖNFELDT, H. C.; GIBSON, N. Changes in the nutrient quality of meat in an obesity context. Meat Science, Barking, v. 80, n. 1, p. 20-27, 2008. http://dx.doi.org/10.1016/j.meatsci.2008.05.025. PMid:22063166.
SPAZIANI, M.; DEL TORRE, M.; STECCHINI, M. L. Changes of physicochemical, microbiological, and textural properties during ripening of Italian low-acid sausages. Proteolysis, sensory and volatile profiles. Meat Science, Barking, v. 81, n. 1, p. 77-85, 2009. http://dx.doi.org/10.1016/j.meatsci.2008.06.017. PMid:22063965.

UYHARA, C. N. S.; OLIVEIRA FILHO, P. R. C.; TRINDADE, M. A.; VIEGAS, E. M. M. Adição de corantes em salsichas de tilápia do Nilo: efeito sobre a aceitação sensorial. Brazilian Journal of Food Technology, Campinas, v. 11, n. 4, p. 271-278, 2008. VOGEL, C. C.; PAZUCH, C. M.; SARMENTO, C.; SECCO, L. B.; HENRIQUE, T. Desenvolvimento de salsicha com teor de sódio reduzido (sal light). Revista Ciências Exatas e Naturais, Guarapuava, v. 13, n. 3, 2011.

VYNCKE, W. Direct determination of the thiobarbituric acid value in trichloroacetic extracts of fish as a measure of oxidative rancidity. Fette-Seinfen Anstrichmittel, Hamburg, v. 72, n. 12, p. 1084-1087, 1970. http://dx.doi.org/10.1002/lipi.19700721218.

YAMADA, E. A.; CIPOLLI, K. M. A. V. B.; HARADA, M. M.; SGARBIERI, V. C. Utilização de extrato de levedura (Saccharomyces sp.) de destilaria de álcool em salsicha. Brazilian Journal of Food Technology, Campinas, v. 13, n. 3, p. 197-204, 2010. http://dx.doi.org/10.4260/BJFT2010130300026.

ZHANG, W.; XIAO, S.; SAMARAWEERA, H.; LEE, E. J.; AHN, D. $U$. Improving functional value of meat products. Meat Science, Barking, v. 86, n. 1, p. 15-31, 2010. http://dx.doi.org/10.1016/j. meatsci.2010.04.018. PMid:20537806. 